\title{
SOBREPESO Y OBESIDAD EN INDÍGENAS NAHUAS DE IXTACZOQUITLÁN, VERACRUZ, MÉXICO
}

\author{
Emma V. Herrera-Huerta1,a,b, Eliud A. García-Montalvo ${ }^{1, a, c}$, Enrique Méndez-Bolaina ${ }^{1, a, d}$, \\ José G. López-López $z^{2, a, b}$, Olga L. Valenzuela ${ }^{1, a, c}$
}

\begin{abstract}
RESUMEN
El estudio tuvo como objetivo determinar la frecuencia de sobrepeso y obesidad en indígenas nahuas de Ixtaczoquitlán, Veracruz, México. Para ello, se realizó un estudio transversal entre los años 2010-2011; donde se calculó el índice de masa corporal (IMC). Para la definición de sobrepeso y obesidad se emplearon las categorías de la Organización Mundial de la Salud (OMS) y los de la Norma Oficial Mexicana (NOM). Se incluyó 227 nahuas (77,5\% mujeres). Según los lineamientos de la OMS, la proporción de nahuas con sobrepeso fue de $41 \%$, y de obesidad $36,5 \%$; y según la NOM fue de 11,4 y $69,2 \%$ respectivamente. En conclusión, la frecuencia de sobrepeso y obesidad en indígenas nahuas es alta. Deben realizarse estudios enfocados a determinar la prevalencia y factores de riesgo y, con ello, desarrollar estrategias de prevención que mejoren la calidad de salud de estas poblaciones.
\end{abstract}

Palabras clave: Sobrepeso; Obesidad; Población indígena; Estado nutricional; México (fuente: DeCS BIREME).

\section{OVERWEIGHT AND OBESITY IN INDIGENOUS NAHUAS FROM IXTACZOQUITLÁN, VERACRUZ, MÉXICO}

\section{ABSTRACT}

\begin{abstract}
The study was aimed at determining the prevalence of overweight and obesity in indigenous nahuas from Ixtaczoquitlán, Veracruz, Mexico. For this purpose, a cross-cut study was conducted between 2010 and 2011, in which the body mass index (BMI) was calculated. To define overweight and obesity, the categories of the World Health Organization (WHO) and the Mexican Official Standard (NOM, Spanish acronym) were used. 227 nahuas (77,5\% women) were included. According to WHO's guidelines, the rate for overweight among nahuas was $41 \%$, and $36.5 \%$ for obesity; according to NOM, it was 11.4 and $69.2 \%$ respectively. In conclusion, the prevalence of overweight and obesity among indigenous nahuas is high. Studies should be conducted to determine the prevalence and risk factors in order to develop prevention strategies based on this information to improve the health quality of these populations.
\end{abstract}

Key words: Overweight; Obesity; Indigenous population; Nutritional status; Mexico (source: MeSH NLM).

\section{INTRODUCCIÓN}

Los cambios sociales y económicos de los últimos años han modificado el estilo de vida de la población y, por ello, se han incrementado los índices de sobrepeso y obesidad a nivel mundial, siendo este un factor de riesgo asociado con enfermedades crónico-degenerativas como diabetes mellitus (DM) tipo 1 y 2, hipercolesterolemia $(\mathrm{HC})$, hipertensión arterial (HTA) e hipertrigliceridemia (HTG), que se han llegado a colocar entre las primeras causas de fallecimiento en México ${ }^{(1-3)}$.
La Organización Mundial de la Salud (OMS) refiere que el $65 \%$ de la población mundial vive en países donde el sobrepeso y la obesidad cobran más vidas de personas que la insuficiencia ponderal (4). En México, la Encuesta Nacional de Salud y Nutrición (ENSANUT 2006) indicó que el sobrepeso y obesidad son problemas que afectan a cerca de $70 \%$ de la población (mujeres, $71,9 \%$; hombres, $66,7 \%$ ) entre los 30 y 60 años, en ambos sexos, siendo la obesidad la que afecta en mayor porcentaje al sexo femenino ${ }^{(5)}$. En Veracruz, la prevalencia del sobrepeso y la obesidad es de $67 \%$ en

Facultad de Ciencias Químicas, Universidad Veracruzana. Veracruz, México

Facultad de Ciencias Químicas, Benemérita Universidad Autónoma de Puebla. Puebla, México

Químico Farmacéutico Biólogo; bdoctor en Farmacia; 'cdoctor en Toxicología; ${ }^{\mathrm{d}}$ doctor en Ciencias

Recibido: 27-04-12 Aprobado: 05-09-12

Citar como: Herrera-Huerta EV, García-Montalvo EA, Méndez-Bolaina E, López-López JG, Valenzuela OL. Sobrepeso y obesidad en indígenas Nahuas de Ixtaczoquitlán, Veracruz, México. Rev Peru Med Exp Salud Publica. 2012;29(3):345-9. 
los adultos mayores de 20 años $(66,6 \%$ para mujeres y $67,6 \%$ para hombres) y $75 \%$ de este grupo de población tiene obesidad abdominal (6).

Pocos estudios se han realizado en poblaciones indígenas de México, algunos de los cuales informan la prevalencia de DM $(4,4 \%)$ en indígenas otomíes de Querétaro (7); en tribus pimas de Sonora (hombres 6,3\% y mujeres $10,5 \%)^{(8)}$; en mazatecas de Oaxaca $(2,1 \%)^{(9)}$, y otros tipos de trastornos metabólicos en poblaciones tepehuana, huichol y mexicanera de Durango(10); mayas de Yucatán ${ }^{(11)}$ y triquis de Oaxaca ${ }^{(12)}$.

Veracruz es una de las once entidades federativas de México donde están concentradas las poblaciones indígenas ${ }^{(13)}$, la mayoría habla dialecto náhuatl. Estos grupos se encuentran aislados de la urbanización conservando rasgos étnicos, conviviendo en zonas eminentemente rurales; sin embargo, la migración ha modificado los hábitos dietéticos con un amplio acceso a alimentos ricos en grasas saturadas y carbohidratos, hallándose en estudios recientes porcentajes significativos de trastornos metabólicos ${ }^{(14)}$. El presente estudio tiene como objetivo aportar en el conocimiento de la frecuencia de sobrepeso y obesidad en una población indígena náhuatl de Ixtaczoquitlán, Veracruz, México.

\section{EL ESTUDIO}

Entre enero de 2010 y mayo de 2011 se realizó un estudio transversal y descriptivo a un grupo de 227 indígenas nahuas, aparentemente sanos, mayores de 18 años, residentes del municipio de Ixtaczoquitlán, en el estado de Veracruz. Se incluyeron indígenas hablantes de náhuatl como primera lengua, con padres y abuelos originarios de esa misma comunidad.

El tamaño de la muestra se calculó considerando una prevalencia del $67 \%$ de sobrepeso y obesidad en el estado de Veracruz ${ }^{(6)}$, un margen de error de seis puntos porcentuales, y un nivel de significancia de $5 \%$, incluyéndose a 227 voluntarios.

Se realizaron las siguientes mediciones: talla, peso y perímetro abdominal. Se calculó el índice de masa corporal (IMC) al dividir el peso $(\mathrm{kg})$ entre la potencia al cuadrado de la talla $\left(\mathrm{m}^{2}\right)$. La clasificación de obesidad se determinó bajo los lineamientos de la Norma Oficial Mexicana NOM-174-SSA1-1998 para el manejo integral de la obesidad (15) determinando obesidad en adultos cuando existe un IMC $\geq 27$, y en población de talla baja $\geq 25$; sobrepeso, cuando el IMC fue $>25$ y $<27$, en población adulta general y $>23$ y $<25$ en población adulta de talla baja. Se consideró talla baja en la mujer adulta si su estatura era menor de 1,50 m y en el hombre si era menor de 1,60 m. También se realizó la clasificación de obesidad según los lineamientos de la de la OMS ${ }^{(16)}$, se determinó obesidad cuando el IMC $\geq 30$ y sobrepeso $\geq 25$. Se midió el perímetro abdominal (PAbd), considerando como "adiposidad abdominal" cuando era $\geq 94 \mathrm{~cm}$ en hombres $\mathrm{y} \geq 80 \mathrm{~cm}$ en mujeres.

Las mediciones fueros registradas por dos observadores capacitados de manera independiente usando las técnicas para las mediciones físicas de la OMS: para la talla se midió (sin zapatos) con un estadímetro, el peso se cuantificó (con ropa ligera y sin zapatos) con una báscula electrónica de litio marca Tylor modelo 7514 (calibrada diariamente); el perímetro abdominal con una cinta métrica convencional (por encima de ropa ligera).

Se analizaron los datos en el programa estadístico STATA 11. El análisis descriptivo consistió en el cálculo de media y desviación estándar en las variables continuas (edad, peso, talla) y de frecuencias simples y proporciones en las variables categóricas (sobrepeso y obesidad).

El estudio fue desarrollado acorde a los lineamientos establecidos por las buenas prácticas clínicas. Se contó con la aprobación del Comité de Ética e Investigación de la Facultad de Ciencias Químicas de la Universidad Veracruzana (FCQ-UV). El consentimiento informado para el reclutamiento de los participantes se basó en los lineamientos de la Ley General de Salud de México y la Declaración de Helsinki.

\section{HALLAZGOS}

El estudio incluyó 227 participantes (22,5\% hombres y $77,5 \%$ mujeres), de 18 a 84 años de edad. El nivel educacional fue bajo $(33,5 \%$ de indígenas $\sin$ instrucción y $58,1 \%$ con estudios de educación básica). El 93,8\% señaló que se dedicaban a actividades del hogar, la agricultura y al comercio informal. La talla y peso promedios fue de $1,49 \pm 0,1 \mathrm{~m}$ y $64,1 \pm 11,0 \mathrm{~kg}$ para las mujeres y 1,57 $\pm 0,1 \mathrm{~m}$ y $71,6 \pm 13,0 \mathrm{~kg}$ para los hombres, respectivamente. La media del IMC medido fue de 28,6 $\pm 5 \mathrm{~kg} / \mathrm{m}^{2}$ (mujeres: 28,6 \pm 5; hombres: 28,7 \pm 4 ). Además, el promedio del perímetro abdominal fue $88,5 \pm 10 \mathrm{~cm}$ (mujeres: 87,6 \pm 10 ; hombres: $91,5 \pm 12$ ) (Tabla 1). Por lo que, la frecuencia de la muestra con adiposidad abdominal fue de $66,4 \%$ (mujeres: $72,6 \%$; hombres: $45 \%$ ).

En la Tabla 2 se observa una diferencia en la frecuencia de sobrepeso más obesidad entre la población estudiada, al emplear los lineamientos de la OMS 
Tabla 1. Características sociodemográficas de la población indígena nahua en estudio del estado de Veracruz, México $(n=227)$.

\begin{tabular}{|c|c|c|c|c|c|c|}
\hline Características & N. ${ }^{\circ}$ & $(\%)$ & Talla (m) & Peso (kg) & $\operatorname{IMC}\left(\mathrm{kg} / \mathrm{m}^{2}\right)$ & PAbd $(\mathrm{cm})$ \\
\hline \multicolumn{7}{|l|}{ Sexo } \\
\hline Mujeres & 176 & $(77,5)$ & $1,49 \pm 0,1$ & $64,1 \pm 11$ & $28,6 \pm 5$ & $87,6 \pm 10$ \\
\hline Hombres & 51 & $(22,5)$ & $1,57 \pm 0,1$ & $71,6 \pm 13$ & $28,7 \pm 4$ & $91,5 \pm 12$ \\
\hline \multicolumn{7}{|l|}{ Edad } \\
\hline $18-39$ & 62 & $(27,3)$ & $1,51 \pm 0,1$ & $65,7 \pm 15$ & $28,4 \pm 6$ & $88,4 \pm 11$ \\
\hline $40-59$ & 105 & $(46,3)$ & $1,51 \pm 0,1$ & $67,4 \pm 11$ & $29,4 \pm 5$ & $90,1 \pm 10$ \\
\hline $60-79$ & 60 & $(26,4)$ & $1,51 \pm 0,1$ & $62,8 \pm 11$ & $27,4 \pm 4$ & $85,9 \pm 10$ \\
\hline \multicolumn{7}{|l|}{ Nivel de educación } \\
\hline Sin instrucción & 76 & $(33,5)$ & $1,51 \pm 0,1$ & $66,1 \pm 11$ & $28,6 \pm 4$ & $88,6 \pm 10$ \\
\hline Primaria & 132 & $(58,1)$ & $1,51 \pm 0,1$ & $65,7 \pm 12$ & $28,6 \pm 5$ & $88,6 \pm 11$ \\
\hline Secundaria & 16 & $(7,1)$ & $1,48 \pm 0,1$ & $63,1 \pm 14$ & $29,0 \pm 7$ & $87,3 \pm 12$ \\
\hline Preparatoria & 3 & $(1,3)$ & $1,52 \pm 0,1$ & $72,0 \pm 26$ & $30,9 \pm 10$ & $90,3 \pm 16$ \\
\hline
\end{tabular}

IMC: Índice de Masa Corporal

PAbd: perímetro abdominal

Nota: se muestra medios \pm desviación estándar.

en comparación con la Norma Oficial Mexicana. La frecuencia de sobrepeso más obesidad calculado según la Norma Oficial Mexicana fue de $80,6 \%$, mientras que el calculado según la OMS fue $77,5 \%$. Sin embargo, la clasificación de obesidad fue mayor al emplear la Norma Oficial Mexicana $(69,2 \%)$ en comparación a la frecuencia obtenida siguiendo los lineamientos de la OMS $(36,5 \%)$.

La frecuencia de sobrepeso (de acuerdo a la OMS) en el grupo etario > 60 años fue mayor $(51,8 \%)$ que en los otros grupos de edad y la proporción de obesidad fue mayor $(53,7 \%)$ en el grupo de $41-50$ años. Cuando las variables de sobrepeso y obesidad se suman el grupo etario que presenta mayor proporción es el de 4150 años con $85,4 \%$ (Tabla 3). En cuanto al sexo, los hombres presentan una frecuencia de sobrepeso más obesidad de $86 \%$, mayor al de las mujeres, en las que es del $75 \%$.

Tabla 2. Comparación de frecuencias de normopeso, sobrepeso y obesidad en la población Nahua de Veracruz, según la clasificación de la OMS y la Norma Oficial Mexicana.

\begin{tabular}{lcr}
\hline & \multicolumn{2}{c}{ Sistema de clasificación } \\
\cline { 2 - 3 } & $\begin{array}{c}\text { OMS } \\
\text { N. }{ }^{\circ}(\%)\end{array}$ & $\begin{array}{c}\text { Norma Oficial } \\
\text { Mexicana } \\
\text { N. } .^{\circ}(\%)\end{array}$ \\
\hline Normopeso & $51(22,5 \%)$ & $44(19,4 \%)$ \\
Sobrepeso & $93(41,0 \%)$ & $26(11,4 \%)$ \\
Obesidad & $83(36,5 \%)$ & $157(69,2 \%)$ \\
$\begin{array}{l}\text { Sobrepeso más } \\
\text { obesidad }\end{array}$ & $176(77,5 \%)$ & $183(80,6 \%)$ \\
\hline
\end{tabular}

OMS: Organización Mundial de la Salud.
Tabla 3. Características de las variables en el grupo indígena Nahua distribuidas por grupo de edad y sexo (de acuerdo con la OMS).

\begin{tabular}{lccccc}
\hline & \multicolumn{5}{c}{ Grupos de edad (años) } \\
\cline { 2 - 6 } & $18-30$ & $31-40$ & $41-50$ & $51-60$ & $>60$ \\
& $\mathrm{~N} .{ }^{\circ}(\%)$ & $\mathrm{N}^{\circ}(\%)$ & $\mathrm{N} .^{\circ}(\%)$ & $\mathrm{N}^{\circ}(\%)$ & $\mathrm{N}^{\circ}(\%)$ \\
\hline Normopeso & $10(35,7)$ & $12(28,6)$ & $6(14,6)$ & $10(16,7)$ & $13(23,2)$ \\
Sobrepeso & $8(28,6)$ & $17(40,5)$ & $13(31,7)$ & $26(43,3)$ & $29(51,8)$ \\
Obesidad & $10(35,7)$ & $13(30,9)$ & $22(53,7)$ & $24(40,0)$ & $14(25,0)$ \\
Sobrepeso & $18(64,3)$ & $30(71,4)$ & $35(85,4)$ & $50(83,3)$ & $43(76,8)$ \\
más obesidad & $18(100)$ & $42(100)$ & $41(100)$ & $60(100)$ & $56(100)$ \\
\hline TOTAL & $28\left(\begin{array}{llll} \\
\hline\end{array}\right.$
\end{tabular}

\section{DISCUSIÓN}

La población de estudio mostró una morfología corporal caracterizada por talla baja (mujeres: 1,49 \pm 0,08 m; hombres: $1,57 \pm 0,10 \mathrm{~m}$ ), variable que fue considerada para el cálculo del IMC bajo los lineamientos de la Norma Oficial Mexicana en comparación con los de la OMS. Donde los resultados difieren debido al ajuste respecto a la talla baja que caracteriza este tipo de poblaciones indígenas del centro y sur de México ${ }^{(11,12)}$. Dado que la obesidad se ha incrementado en todo el mundo, es necesario particularizar ciertas poblaciones, como las indígenas de México, ya que por sus características étnicas se sobreestimarían las frecuencias de sobrepeso y obesidad en la población con talla baja ${ }^{(17)}$, premisa que también ha sido puntualizada en el estudio de Arroyo et al. ${ }^{(11)}$ en población maya.

Existe una diferencia de $29,6 \%$ en las frecuencias halladas de sobrepeso calculado por la OMS y por la norma mexicana, así como la variable obesidad con un $32,7 \%$ de diferencia. Es importante resaltar estas 
diferencias, ya que dependiendo de la referencia aplicada podríamos realizar una inadecuada comparación en la clasificación del IMC, por ello es conveniente mostrar ambas para poblaciones de México. Esta diferencia en el cálculo se debe a que las poblaciones indígenas en México se caracterizan por su talla baja ${ }^{(11,12)}$, por lo que las medidas de salud pública deben ser acordes a ello.

La frecuencia de las variables sobrepeso más obesidad (según la OMS) fue $77,5 \%$, este resultado es $7,5 \%$ superior al obtenido en la ENSANUT 2006 (70\%) ${ }^{(5)}$ que es la encuesta de referencia a nivel nacional; y es similar a un estudio realizado en mayas (grupo indígena de Yucatán, México $)^{(11)}$ quienes presentaron una prevalencia de sobrepeso más obesidad del 78,6\%; mientras que el resultado obtenido en el presente estudio en población indígena Nahua mediante la norma mexicana fue $80,6 \%$, valor $3,1 \%$ mayor que el de la OMS.

También, la frecuencia de adiposidad abdominal hallado en la población de estudio fue de 66,4\%, que es 10\% menor al descrito en la ENSANUT 2006 para la población general de México $(75,9 \%){ }^{(5)}$, para el estado de Veracruz $(75 \%)^{(6)}$ o por los mayas de Yucatán $(75 \%)^{(11)}$. Siendo el sexo femenino el de mayor frecuencia en todos los estudios.

El grupo de 41 a 50 años tuvo la mayor frecuencia de obesidad $(53,7 \%)$ en comparación a los demás grupos de edad. Es probable que la elevada frecuencia de obesidad en este grupo esté relacionada con la falta de actividad física y al alto consumo de carbohidratos en la dieta, debido a una inadecuada alimentación, ya que la mayoría de las mujeres de este grupo de edad se dedican al hogar, mientras que los hombres dedican menos tiempo a las actividades agrícolas intensas, lo cual se ve reflejado en la alta frecuencia de sobrepeso y obesidad en el sexo masculino.

Las limitaciones del estudio fueron que las medidas antropométricas y la aplicación de encuestas se obtuvieron solo una vez y, por lo tanto, no fue posible realizar un seguimiento del incremento del peso en el transcurso del tiempo, pero esto se considerará en un próximo estudio. Asimismo, cabe la posibilidad de que un gran número de mujeres accediera a participar en el estudio debido a que se dedican a las labores del hogar, mientras que los hombres no tienen el tiempo para participar en los estudios, y porque pueden tener un menor interés en su estado de salud. No debe olvidarse que los resultados obtenidos provienen de datos agregados tras utilizar como unidad de análisis la población indígena de Ixtaczoquitlán, Veracruz. Por todo ello, los resultados aquí mostrados solo podrían ser extrapolados a este nivel de agrupación teniendo en cuenta un posible sesgo de selección de la población, con preferencia del grupo femenino.

Este tipo de estudios, como muchos otros, deja al descubierto que los pueblos indígenas presentan rezagos y desigualdades en los servicios de salud (18), este problema también se presenta en otros países en los cuales se detectan ciertas barreras de acceso a los servicios de salud debido a su origen étnico ${ }^{(19,20)}$. La Organización Panamericana de la Salud (OPS) indica que la obesidad en una condición prevenible a través de acciones poblacionales e individuales. Por ello deben analizarse con más profundidad este tipo de poblaciones vulnerables y detectar aquellos factores que las alejan de los servicios de salud y por consiguiente proponer estrategias que mejoren la calidad de vida de los indígenas en México y del mundo ${ }^{(21)}$.

En conclusión, la frecuencia de sobrepeso y obesidad en indígenas nahuas es alta, aunque diferenciada según el sistema de clasificación que se emplee (el de la OMS o el de la Norma Oficial Mexicana). Deben realizarse estudios enfocados a determinar la prevalencia y factores de riesgo y con ello desarrollar estrategias de prevención que mejoren la calidad de salud de estas poblaciones.

Contribuciones de autoría: EVHH es la responsable directa del proyecto. OLVL realizó la asesoría estadística. EMB y JGLL participaron en la revisión crítica del artículo y asesoría técnica. Todos los autores participaron en la concepción y diseño del estudio; obtención, análisis e interpretación de datos, redacción del artículo y en la aprobación de la versión final del artículo.

Fuentes de financiamiento: Secretaría de Educación Pública, Programa PROMEP, Referencia del Proyecto PROMEP-UVPTC-209.

Conflictos de interés: los autores declaran no tener conflictos de interés. 


\section{REFERENCIAS BIBLIOGRÁFICAS}

1. Rojas R, Aguilar-Salinas CA, JiménezCorona A, Shama-Levy T, Rauda J, ÁvilaBurgos L, et al. Metabolic syndrome in Mexican adults. Results from the National Health and Nutrition Survey 2006. Salud Publica Mex. 2010;52 suppl 1:S11-8.

2. Jiménez-Corona $A$, Rojas $R$, Gómez-Pérez FJ, Aguilar-Salinas CA. Early-onset type 2 diabetes in a Mexican survey: Results from the National Health and Nutrition Survey 2006. Salud Publica Mex. 2010;52 suppl $1: S 27-35$.

3. Barquera $S$, Campos-Nonato I, HernándezBarreraL,VillalpandoS, Rodríguez-Gilabert $\mathrm{C}$, Durazo-Arvizú R, et al. Hypertension in Mexican adults: results from the National Health and Nutrition Survey 2006. Salud Publica Mex. 2010;52 suppl 1:S63-71.

4. World Health Organization. Obesity and overweight [Internet]. Geneva: WHO; 2011 [citado el 12 septiembre 2011]. Disponible en: http://www.who.int/ mediacentre/factsheets/fs311/en/index. html

5. Olaiz-Fernández G, Rivera-Dommarco J, Shamah-Levy T, Rojas R, VillalpandoHernándezS, Hernández-Avila M, et al (ed). Encuesta Nacional de Salud y Nutrición 2006. Cuernavaca, México: Instituto Nacional de Salud Pública, 2006.

6. Instituto Nacional de Salud Pública. Encuesta Nacional de Salud y Nutrición 2006. Resultados por entidad federativa, Veracruz. Cuernavaca, México: Instituto Nacional de Salud Pública-Secretaría de Salud, 2007.

7. Alvarado-Osuna C, Milian-Suazo F, VallesSánchez V. Prevalencia de diabetes mellituse hiperlipidemias en indígenas otomíes. Salud Publica Mex. 2001;43(5):459-63.
8. Ravussin E, Valencia ME, Esparza J, Bennett PH, Schulz LO. Effects of a traditional lifestyle on obesitiy in Pima Indians. Diabetes Care. 1994;17(9):1067-74.

9. Castro S, Escobedo P. La prevalencia de la diabetes mellitus no dependiente de insulina y factores de riesgo asociados, en población mazateca del estado de Oaxaca, México. Gac Med Mex. 1997;133(6):527-34.

10. Guerrero-Romero F, Rodríguez-Morán M, Sandoval-Herrera F. Low prevalence of noninsulin-dependent diabetes mellitus in indigenous communities of Durango, Mexico. Arch Med Res. 1997;28(1):13740.

11. Arroyo P, Fernández V, Loría A, Pardío J, Laviada H, Vargas-AnconaL, et al. Obesidad, morfología corporal y presión arterial en grupos urbanos y rurales de Yucatán. Salud Publica Mex. 2007;49(4):274-85.

12. Ramos-Rodríguez RM, Sandoval-Mendoza K. Estado nutricional en la marginación y la pobreza de adultos triquis del estado de Oaxaca, México. Rev Panam Salud Pública. 2007;22(4):206-7.

13. Comisión Nacional para el Desarrollo de los Pueblos Indígenas [Internet]. México: Los pueblos indígenas de México; 2008 [citado el 12 septiembre 2011]. Disponible en: http://www.cdi.gob.mx

14. Manzúr-Mejía MA. Evaluación de las dislipidemias y su relación con la etnicidad de una población rural del Estado de Veracruz [Tesis de Licenciatura]. Orizaba, Veracruz: Facultad de Ciencias Químicas, Universidad Veracruzana; 2009.

15. México, Secretaría de Salud. Norma Oficial Mexicana NOM-174-SSA1-1998, Para el manejo integral de la obesidad [Internet]. México: Secretaria de Salud; 2012.
16. World Health Organization. Obesity: Preventing and managing the global epidemic. Report of a WHO Consultation. Geneva: World Health Organization; 2000.

17. García-García E, De la Llata-Romero M, Kaufer-Horwitz M, Tusié-Luna MT, Calzada-León R, Vázquez-Velázquez V, et al. La obesidad y el síndrome metabólico como problema de salud pública. Una reflexión. Salud Publica Mex. 2008;50(6):530-47.

18. PelcastreB.Lasalud delos pueblos indígenas: acciones y políticas. Mesa redonda IX. Salud Publica Mex. 2007;49:83.

19. Hautecoeur M,Zunzunegui MV,Vissandjee B. Las barreras de acceso a los servicios de salud en la población indígena de Rabinal en Guatemala. Salud Publica Mex. 2007;49(2):86-93.

20. Bustos P, Muñoz S, Vargas C, Amigo H. Pobreza y procedencia indígena como factores de riesgo de problemas nutricionales de los niños que ingresan a la escuela. Salud Publica Mex. 2009;51(3):187-93.

21. Organización Panamericana de la Salud. Métodos para la prevención y el control de la obesidad y de la diabetes. Región de las Américas. Epidemiological Bulletin [Internet]. 2008 [citado el 5 de agosto de 2012];27(2):[aprox 6 p.] Disponible en: http://new.paho.org/bulletins/index. php?option=com_content\&task=view\&id $=195 \&$ Itemid $=272$

Correspondencia: Emma V. Herrera-Huerta Dirección: Prol. Ote. 6, No.1009, Col. Rafael Alvarado. C.P. 94340 Orizaba, Veracruz, México. Teléfono: (0052) 2727240120

Correoelectrónico:emherrera@uv.mx

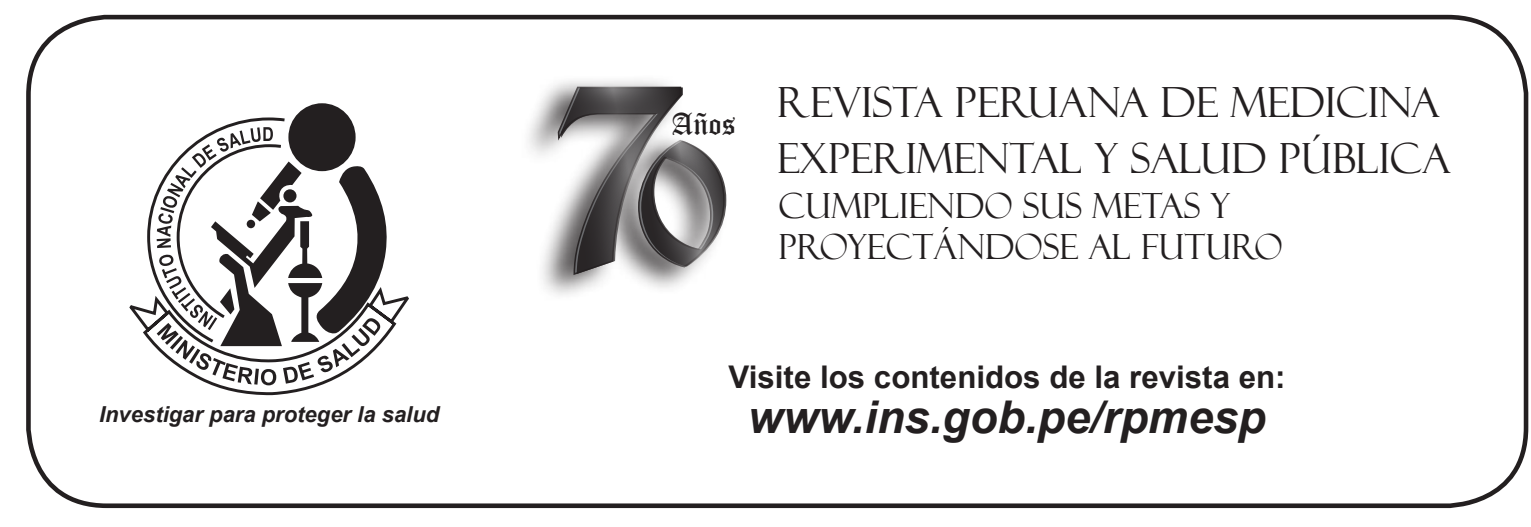

